\title{
EFFECTS OF INTRACLASS CORRELATION ON WEIGHTED AVERAGES 1 '
}

\author{
Shayle R. Searle* and Friedrich Pukelsheim \\ Institut fir Mathematik \\ Universität Augsburg, Federal Republic of Germany
}

$B U-881-m$

ABSTRACT
Weighted averages of class means using different sets of weighting
factors are compared in terms of sampling variances and of relative weights
given to the class means. Details are given for the 1-way classification,
and extensions to other models are indicated.

\section{INTRODUCTION}

When subclasses of data have unequal numbers of observations, averages of the subclass means can be defined in a variety of ways, depending on the weights used for (linearly) combining the subclass means. At least three different weighting systems are often used: (I) weighting by the number of observations, which leads to the grand mean; (II), weighting equally, which yields the simple average of the subclass means, and (III), weighting inversely according to variances of the observed subclass means. In the 1-way classification, with the fixed effects model, III is the same as II; but with the random effects model (which we call the mixed model, see Section 3.1) in which the class effects are taken as random, I and II are special cases of III corresponding to the intra-class correlation being 0 and 1 , respectively.

\footnotetext{
*on leave from Cornell University, Ithaca, N.Y., supported by a U. S. Sentor Scientist Award from the Alexander von Humboldt-Stiftung.

$1 /$ Paper number BU-477 in the Biometrics Unit, Cornell University, Ithaca, N.Y.
} 
Variances of these weighted averages are compared, in both models, and the manner in which changes in the intra-class correlation affects the relative weights given to the class means is described. Extensions to 2-way classifications are suggested.

\section{FIXED EFFECTS MODELS}

\subsection{A model}

Suppose $y_{1 j}$ is the $j$ 'th observation of the $i$ th class of a 1 -way classification, with $1=1, \cdots$, and $j=1, \cdots, n_{i} ; 1 . e .$, a classes and $n_{1}$ observations in the $i^{\prime}$ th class. Then the model equation for $y_{1 j}$ can be taken as

$$
y_{i j}=\mu+\alpha_{i}+e_{i j}=\mu_{i}+e_{i j}
$$

in which $\mu_{i}=\mu+\alpha_{i}$ is the population mean of the $i$ th class, and the $e_{i j}$ terms are random variables, identically distributed with zero mean, variance $\sigma_{e}^{2}$, and zero covariances. Under these conditions the BLUE (best linear unbiased estimator) of $\mu_{1}$ and the sampling variance of that estimator are, respectively,

$$
\hat{\mu}_{i}=\bar{y}_{i}=\sum_{j=1}^{n} y_{1 j} / n_{i} \text { and } v_{F}\left(\bar{y}_{i}\right)=\sigma^{2} / n_{i} \text {, }
$$

similar to Searle (1971, pages 235 and 339$)$. In (2) the subscript F in $v_{F}\left(\bar{y}_{1}\right)$ emphasizes that the variance is based on the fixed effects model.

\subsection{Weighted Averages}

We begin with weighted averages I and II of the introduction. The first is denoted $\mu_{n}$, in which weights proportional to the numbers of observations are used:

$$
\mu_{n}=\sum n_{1} \mu_{1} / \sum n_{i}
$$

(All summations are with respect to $i$, over the range $i=1,2, \cdots, a$.$) The$ second weighted average is denoted $\mu_{e}$, and is based on equal weights:

$$
\mu_{e}=\sum \mu_{1} / a
$$


The third average mentioned in the introduction uses weights inversely proportional to $v\left(\bar{y}_{1}\right)$ and so, on using (2), is the same as $\mu_{n}$ :

$$
\sum\left(\mu_{1} n_{1} / \sigma_{e}^{2}\right) / \sum\left(n_{1} / \sigma_{e}^{2}\right)=\sum n_{1} \mu_{i} / \sum n_{i}=\mu_{n}
$$

A quite general form of weighted average is to use arbitrary, (usually) positive weights $w_{1}$ :

$$
\mu_{w}=\sum w_{i} \mu_{i} / \sum w_{i} .
$$

Then $\mu_{n}$ and $\mu_{e}$ are special cases of $\mu_{w}$, since $w_{i}=n_{i}$ gives $\mu_{w}=\mu_{n}$, and $w_{i}=1$ gives $\mu_{w}=\mu_{e}$. The BLUEs of these three averages and their sampling vartances are

$$
\begin{array}{ll}
\hat{\mu}_{n}=\sum n_{i} \bar{y}_{i} / \sum n_{i}=\bar{y}_{\ldots}, & \text { with } v_{F}\left(\hat{\mu}_{n}\right)=\sigma_{e}^{2} / \sum n_{i}, \\
\hat{\mu}_{e}=\sum \bar{y}_{i} / a, & \text { with } v_{F}\left(\hat{\mu}_{e}\right)=\sigma_{e}^{2}\left(\sum 1 / n_{i}\right) / a^{2} \\
\hat{\mu}_{w}=\sum w_{i} \bar{y}_{i} / \sum w_{i}, \ldots \quad \text { with } v_{F}\left(\hat{\mu}_{w}\right)=\sigma_{e}^{2}\left(\sum w_{i}^{2} / n_{i}\right) /\left(\sum w_{i}\right)^{2} .
\end{array}
$$

Clearly, $\hat{\mu}_{n}$ is the grand mean $\bar{y}_{.}$, whereas $\hat{\mu}_{e}$ is the average of observed class means, $\sum \bar{y}_{1} / a$.

\subsection{Discussion}

Estimators (3), (4) and (5) are BLUEs of different parametric functions, so that comparing their sampling variances does not seem, a priori, to be beneficial. However, in Section 3 we are interested in the case in which the subclass means $\mu_{1}$ are all the same, namely $\mu$, whereupon the three estimators all estimate $\mu$. Comparing variances of those estimators is then of interest. As prelude to this, the variances in (3) - (5) are compared, beginning with those of $\hat{\mu}_{n}$ and $\hat{\mu}_{w}$.

From applying the Cauchy-Schwartz inequality, $\sum \mathrm{p}^{2} \sum \mathrm{q}^{2} \geq\left(\sum \mathrm{pq}\right)^{2}$, we have

$$
\sum n_{1} \sum w_{1}^{2} / n_{1} \geq\left(\sum \sqrt{n_{1} w_{1}^{2} / n_{1}}\right)^{2}=\left(\sum w_{1}\right)^{2}
$$


Hence

$$
1 / \sum n_{1} \leq\left(\sum w_{1}^{2} / n_{1}\right) /\left(\sum w_{1}\right)^{2}
$$

and so from (3) and (5)

$$
v_{F}\left(\hat{\mu}_{n}\right) \leq v_{F}\left(\hat{\mu}_{w}\right)
$$

Therefore, in the fixed effects model, no weighted average of the $\mu_{1} s$ has a BLUE with variance smaller than that of $\hat{\mu}_{n}$. This is an attractive property for $\hat{\mu}_{n}$. In particular, it applies for $w_{1}=1$, giving

$$
v_{F}\left(\hat{\mu}_{n}\right) \leq v_{F}\left(\hat{\mu}_{e}\right)
$$

This is perhaps a little surprising, since defining an overall mean as $\mu_{e}$ seems more natural than does $\mu_{n}$ because of the dependence of $\mu_{n}$ on the numbers of observations in the classes.

In applications, $\mu_{w}$ for a particular set of $w_{i}$-values can well be a parameter of interest; e.g., if three varieties of wheat are grown in a county in acreages proportional to $w_{1}: w_{2}: w_{3}$, the county's mean wheat yield per acre is $\mu_{w}$. Therefore, if in some experiment designed to measure yield the areas in which the three varieties are grown are proportional to $n_{1}: n_{2}: n_{3}$, different from $w_{1}: w_{2}: w_{3}$, then $\mu_{n} \neq \mu_{w}$, and $\hat{\mu}_{w}$ will be the estimated mean of interest. Nevertheless, (6) shows that $\hat{\mu}_{w}$ always has variance greater (strictly, never less) than that of $\hat{\mu}_{n}$. This suggests one reason for having subclass sizes in data proportional to subclasses population sizes.

\section{MIXED MODELS}

\subsection{A model}

Suppose with the model equation (1) we take the $\alpha_{1} s$ as uncorrelated random effects with zero means and variance $\sigma_{\alpha}^{2}$, with the covariance between every $\alpha_{i}$ and every $e_{h k}$ being zero. The $e_{1 j}$ terms retain the same mean, variance and covarlance properties as described following (1). With these properties, the model is usually called the random effects model, or 
random model, of the 1-way clasification. But since $\mu$ is a fixed effect and the $\alpha_{1} s$ are random effects it is strictly a mixed model, and we think of it in that manner for purposes of estimating $\mu$ in the presence of the random effects.

\subsection{Weighted averages and estimators.}

In the preceding mixed model the BLUE of $\mu$, to be denoted $\hat{\mu}_{r}$ is, simflar to Searle (1971, page 463),

$$
\hat{\mu}_{r}=\sum \frac{n_{i}}{n_{i} \sigma_{\alpha}^{2}+\sigma_{e}^{2}} \bar{y}_{i} / \sum \frac{n_{i}}{n_{i} \sigma_{\alpha}^{2}+\sigma_{e}^{2}} \text { with } v_{M}\left(\hat{\mu}_{r}\right)=1 / \sum \frac{n_{i}}{n_{i} \sigma_{\alpha}^{2}+\sigma_{e}^{2}} \text {. }
$$

The subscript $M$ in $v_{M}$ of (7) denotes variance based on the mixed model. The estimator $\hat{\mu}_{r}$ in (7) is, of course, a special case of $\hat{\mu}_{w}$ with $w_{i}=n_{i} /\left(n_{i} \sigma_{\alpha}^{2}+\sigma_{e}^{2}\right)$; and if $\hat{\mu}_{w}$ for other values of $w_{i}$ is to be used, its variance is

$$
v_{M}\left(\hat{\mu}_{w}\right)=\frac{\sum w_{i}^{2}\left(\sigma_{\alpha}^{2}+\sigma_{e}^{2} / n_{i}\right)}{\left(\sum w_{i}\right)^{2}}
$$

derived by replacing $\sigma_{e}^{2} / n_{1}$ in $v_{F}\left(\hat{\mu}_{w}\right)$ of (5) with $\sigma_{\alpha}^{2}+\sigma_{e}^{2} / n_{i}$.

\section{3 Comparing variances of estimated averages}

First, from (8) and (5) it is easily seen that

$$
v_{M}\left(\hat{\mu}_{w}\right)=\sigma_{\alpha}^{2} \frac{\sum w_{i}^{2}}{\left(\sum w_{1}\right)^{2}}+v_{F}\left(\hat{\mu}_{w}\right)>v_{F}\left(\hat{\mu}_{w}\right) \text { for } \sigma_{\alpha}^{2}>0 .
$$

Thus every weighted average has variance in the mixed model that exceeds its variance in the fixed model - as one would expect. (When $\sigma_{\alpha}^{2}=0$ the variances are equal.) What is more interesting 1 s that by applying the same reasoning to (7) and ( 8 ) as is used in deriving (6), it is easily shown that

$$
v_{M}\left(\hat{\mu}_{r}\right) \leq v_{M}\left(\hat{\mu}_{w}\right)
$$


This shows that in the mixed model no weighted average $\hat{\mu}_{w}$ has smaller variance than does $\hat{\mu}_{r}$ (as 18 to be expected because $\hat{\mu}_{I}$ is the BLUE of $\mu$ ). A special case of (9) is $v_{M}\left(\hat{\mu}_{r}\right) \leq v_{M}\left(\hat{\mu}_{n}\right)$. Nevertheless, $v_{F}\left(\hat{\mu}_{n}\right)$ of (3) is less than $v_{M}\left(\hat{\mu}_{r}\right)$ of (7), as may be seen by observing that

$$
1 / v_{F}\left(\hat{\mu}_{n}\right)-1 / v_{M}\left(\hat{\mu}_{r}\right)=\sum n_{1}\left[1 / \sigma_{e}^{2}-1 /\left(n_{1} \sigma_{\alpha}^{2}+\sigma_{e}^{2}\right)\right]>0 \text {, for } \sigma_{\alpha}^{2}>0 .
$$

Hence

$$
v_{F}\left(\hat{\mu}_{n}\right) \leq v_{M}\left(\hat{\mu}_{r}\right) \leq v_{M}\left(\hat{\mu}_{n}\right)
$$

Thus the variance of $\hat{\mu}_{r}$ in the mixed model is between that of $\hat{\mu}_{n}$ in the fixed and mixed models, with these variances being equal when $\sigma_{\alpha}^{2}=0$, for then $\hat{\mu}_{\mathrm{n}}=\hat{\mu}_{\mathrm{r}}$.

\subsection{Relative weights for observed subclass weans in $\hat{\mu}_{r}$}

In $\hat{\mu}_{n}$ the observed subclass means, $\bar{y}_{i}$, are weighted in proportion to their $n_{i}$-values; and in $\hat{\mu}_{e}$ they are welghted equally. In the mixed model with intra-class correlation $\rho=\sigma_{\alpha}^{2} /\left(\sigma_{\alpha}^{2}+\sigma_{e}^{2}\right)$, it is interesting to see how the weights in $\hat{\mu}_{r}$ change from those of $\hat{\mu}_{n}$ when $\rho=0$ to those of $\hat{\mu}_{e}$ when $\rho=1$. To observe this, write $\hat{\mu}_{r}$ of (7) as

$$
\hat{\mu}_{r, \rho}=\sum \frac{n_{i}}{n_{i}^{\rho}+1-\rho} \bar{y}_{i} / \sum \frac{n_{i}}{n_{i} \rho+1-\rho} .
$$

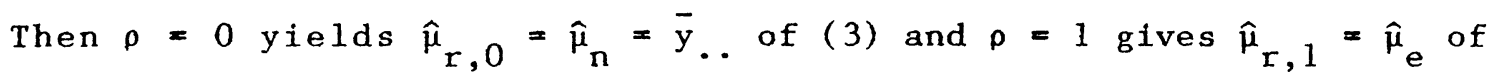
(4). This is not surprising. $\rho=0$ is equivalent to $\sigma_{\alpha}^{2}=0$ which reduces the mixed model to being a fixed effects model $y_{1 j}=\mu+e_{1 j}$ and so $\hat{\mu}_{r, 0}=$ $\hat{\mu}_{n}$, the BLUE of $\mu$ in that model. And $\rho=1$, although equivalent to $\sigma_{e}^{2}=0$, is more interestingly the case of observations within each class being perfectly correlated - In effect, identical. Hence, no matter what the value of $n_{1}$ is, $\bar{y}_{1}$ has variance $\sigma_{\alpha}^{2}$ and so the linear combination of $\bar{y}_{i} s$ that has minimum variance is $\hat{\mu}_{e}=\sum \bar{y}_{i} / a$. 
Despite these consequences of putting $\rho=0$ and $\rho=1$ in $\hat{\mu}_{I}$, it $1 \mathrm{~s}$ nevertheless surprising how quickly the weights given to each $\bar{y}_{1}$ change from being proportional to $n_{1}$ in $\hat{\mu}_{r, 0}=\hat{\mu}_{n}$ to approaching being equal in $\hat{\mu}_{r, 1}=\hat{\mu}_{e}$ as $p$ increases from 0 to 1 . Consider two classes, one described as having a large number of observations, $n_{L}$, and the other having a small number, $n_{S}$, with of course, $n_{L}>n_{S}$. In $\hat{\mu}_{r}$ the ratio of the weight given $\bar{y}_{S}$ to that given to $\bar{y}_{L}$ is $\tau_{\rho}$ say, where, from (11),

$$
\tau_{\rho}=\frac{\text { coefficient of } \bar{y}_{S} \text { in } \hat{\mu}_{T, \rho}}{\text { coefficient of } \bar{y}_{L} \text { in } \hat{\mu}_{T, \rho}}=\frac{n_{S}\left(n_{L} \rho+1-\rho\right)}{n_{L}\left(n_{S} \rho+1-\rho\right)}=\frac{\rho+\frac{1-\rho}{n_{L}}}{\rho+\frac{1-\rho}{n_{S}}} \text {. }
$$

Corresponding to $\rho=0$ with $\hat{\mu}_{r, 0}=\hat{\mu}_{n}$ we have $\tau_{0}=n_{S} / n_{L} ;$ and as $\rho$ Increases from zero to unity $\tau_{\rho}$ increases from $\tau_{0}=n_{S} / n_{L}$ to $\tau_{1}=1$. Thus as $p \rightarrow 1$ we see that $\bar{y}_{S}$, the data mean of the smaller-sized class, gets increasingly larger weights in $\hat{\mu}_{r, \rho}$, relative to $\bar{y}_{L}$. It is interesting to see that this increase can, depending on the magnitudes of $n_{L}$ and $n_{S}$, be quite appreciable, even for very small values of $\rho$. This is so because the first derivative of $\tau_{p}$ with respect to $\rho$ is

$$
\tau_{\rho}^{\prime}=\frac{\partial \tau_{\rho}}{\partial \rho}=\left(\frac{1}{n_{S}}-\frac{1}{n_{L}}\right) /\left(\rho+\frac{1-\rho}{n_{S}}\right)^{2},
$$

and for small values of $\rho$ and not-too-small values of $n_{S}$ this can be relatively large. In particular, for $\rho=0$

$$
\tau_{0}^{\prime}=n_{S}\left(1-\frac{n_{S}}{n_{L}}\right)
$$

and so when $n_{S} / n_{L}$ is small and $n_{S}$ is not too small, $\tau_{0}^{\prime}$ can be relatively large: e.g., for $n_{S}=20$ and $n_{L}=100, \tau_{0}^{\prime}=20(1-0.2)=16$. This 1 s the slope at $\rho=0$ of $\tau_{\rho}$ plotted against $\rho$. The value 16 represents an angle 
of $86.4^{\circ}$ from the horizontal, which means that, for values of $p$ near zero, $\tau_{p}$ increases very rapidly from $\tau_{0}=n_{S} / n_{L}=20 / 100=.2$. This is evident in the second column of the following table, which shows values of $\tau_{p}$ for three pairs of $n_{S}, n_{L}$ values and a range of values of $p$.

DEPENDENCE ON INTRA-CLASS CORRELATION OF THE RELATIVE WEIGHTS GIVEN TO TWO OBSERVED SUBCLASS MEANS IN THE ESTIMATOR

$$
\hat{\mu}_{r ; \rho}=\sum \frac{n_{1}}{n_{i} \rho+1-\rho} \bar{y}_{i} / \sum \frac{n_{i}}{n_{i} \rho+1-\rho} .
$$

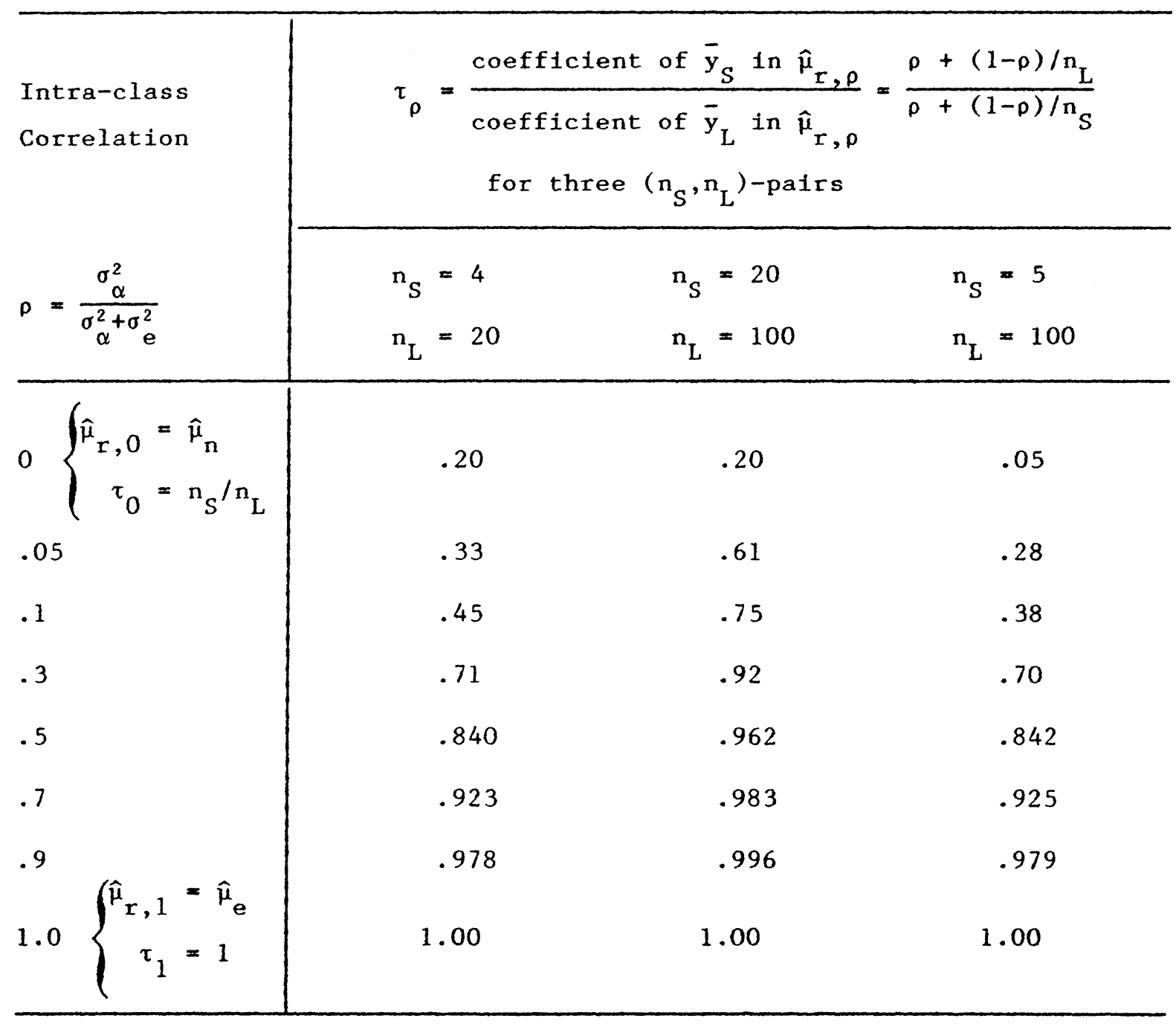




\subsection{Discussion}

The BLUE of $\mu$ in the mixed model is $\hat{\mu}_{r} ; 1$ it reduces to $\hat{\mu}_{n}=\bar{y}_{\text {. . in }}$ the fixed model wherein $\sigma_{\alpha}^{2}=0$, and to $\hat{\mu}_{e}=\Sigma \bar{y}_{1} / a$ in the trivial case of $\sigma_{e}^{2}=0$ when all observations in each class are then identical; and, of course, if every $n_{i}$ has the same value then $\hat{\mu}_{n}=\hat{\mu}_{e}=\bar{y}_{.}$. Each of the estimators $\hat{\mu}_{n}, \hat{\mu}_{e}$ and $\hat{\mu}_{r}$ has variance in the mixed model that exceeds its variance in the fixed model, as is, of course, to be expected. However, as in (9), in the mixed model $\hat{\mu}_{r}$ has the smallest variance of any (linearly) weighted average although in the fixed model $\hat{\mu}_{n}$ has still smaller variance.

In $\hat{\mu}_{r}$ the weight given to $\bar{y}_{S}$ having $n_{S}$ observations, relative to that given to $y_{L}$ with $n_{L}>n_{S}$ observations, is $\tau_{\rho}$ given by (12). The value of $\tau_{p}$ is $n_{S} / n_{L}$ for $\rho=0$, i.e., in $\hat{\mu}_{n}$; and $i t$ is 1.0 for $\rho=1$, i.e., in $\hat{\mu}_{\mathrm{e}}$. The rate of increase in $\tau_{\mathrm{p}}$ for $\rho$ increasing from 0 to 1 is given by $\tau_{\rho}^{\prime}$ of (13) with $\tau_{0}^{\prime}=n_{S}\left(1-n_{S} / n_{L}\right)$. Thus for small values of $\rho$ the rate of increase in $\tau_{p}$ depends not only on $n_{S} / n_{L}$ but also on $n_{S}$; hence small changes in $\rho$ can bring about big changes in $\tau_{\rho}$. This is illustrated in the table where, for the example having $n_{S}=20$ and $n_{L}=100$, changing $\rho$ from 0 to .05 changes $\tau_{p}$ from .20 to .61 . Thus not only can relative sizes of data subclasses be important in the contributions that observed subclass means make to $\hat{\mu}_{r}$, but absolute sizes are also important. This is also illustrated in the table, where in each of the first two examples $n_{S} / n_{L}=.2:$ in the first of these, $n_{S}=4$ and $\tau_{.05}$ is 0.33 , whereas in the second, with $n_{S}=20$ the value of $\tau_{.05}$ is .61 , nearly double its value for $\mathrm{n}_{\mathrm{S}}=4$. 


\section{EXTENSIONS}

Consider a 2-way nested classification where the number of main classes is a, with the $I^{\prime}$ th having b, subclasses, in the $f$ th of which there are $n_{i j}$ observations $y_{i j k}$ for $k=1, \cdots, n_{i j}$, with $1=1, \cdots, a$ and $f=1, \cdots, b_{i}$ A mixed model for this situation can be taken as $y_{i j k}=\mu_{i}+\beta_{1 j}+e_{i j k}$ with $\mu_{1}$ as a fixed effect and $\beta_{i j}$ and $e_{1 j k}$ as random effects with zero means, variances $\sigma_{B}^{2}$ and $\sigma_{e}^{2}$, respectively, and with all covariances zero. Then, similar to $\hat{\mu}_{r}$ of $(7)$, the BLUE of $\mu_{i}$ is

$$
\hat{\mu}_{i}=\sum_{j=1}^{b} \frac{n_{i j}}{n_{i j} \sigma_{\beta}^{2}+\sigma_{e}^{2}} \bar{y}_{i j}, \quad, \sum_{j=1}^{b} \frac{n_{i j}}{n_{i j} \sigma_{\beta}^{2}+\sigma_{e}^{2}} .
$$

Discussions of this and of linear combinations of the $\hat{\mu}_{i} s$, can be made similar to those of Sections 2 and 3 . Analogous extensions could also be made for a 2-way crossed classification for combining BLUEs $\hat{\mu}_{i j}=\vec{y}_{i j}$. in situations where $v\left(\bar{y}_{i j \cdot}\right)=\sigma_{\gamma}^{2}+\sigma_{e}^{2} / n_{1 j}$.

\section{REFERENCES}

Searle, S. R. (1971) Linear Models, Wiley, New York. 